\title{
Experiencia de un año de hemodiafiltración "on-line" en pacientes tratados previamente con hemodiálisis convencional
}

\author{
Antonia Freile Solsona - Carme Sanchez Fernandez - Sebastia Pujol i Massa
}

Centre de Dialisi Mataró. Diaverum Catalunya

\section{Sra. Directora:}

En los últimos años el tratamiento de hemodiálisis ha sufrido una rápida y notable evolución. Nuestros monitores han dejado de ser simples lectores de presiones y disponemos de monitores con capacidad de auto regulación. Sin duda, hemos iniciado un camino que nos conduce a técnicas cada vez mas sofisticadas y precisas cuya finalidad última es una mejora en la calidad asistencial para nuestros pacientes, lo que nos debería trasladar a una mejor calidad de vida y mayor supervivencia ${ }^{1-3}$. En este camino estamos empezando a sustituir de forma más o menos generalizada, las técnicas hemodialíticas convencionales por otras como la hemodiafiltración en línea.

Realizamos un estudio comparativo en los pacientes de nuestro centro sometidos a ambas tratamientos, aportando a los numerosos estudios realizados, nuestra particular experiencia, planteándose los siguientes objetivos:

- Evaluar la mejor calidad de diálisis mediante el estudio de las diferencias en parámetros analíti$\cos (\mathrm{Kt} / \mathrm{v}, \mathrm{Hb})$, y requerimientos de eritropoyetina.

- Evaluar subjetivamente la percepción de una mejor calidad de vida en dichos pacientes.

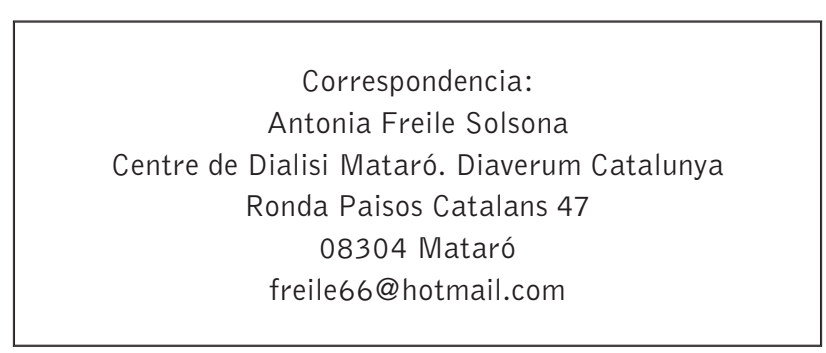

Este estudio retrospectivo, abarca dos años, el anterior al inicio a la hemodiafiltración en línea, y el primer año de inicio de esta técnica. Se seleccionaron a los pacientes que hubieran estado sometidos anteriormente a hemodiálisis convencional de alto flujo (HDAF), estableciendo un período mínimo de un año y sin máximos retrospectivos, obteniéndose una media para el grupo de 48 meses de terapia en HDAF. Y al mismo tiempo, que estos mismos pacientes llevaran más de 11 meses tratados con hemodiafiltración en línea postdilución (HDFPO).

El estudio se realiza a 21 pacientes ( 15 hombres, 6 mujeres) en tratamiento con hemodiafiltración en línea postdilución (HDFPO), con edad comprendida entre los 34 y 87 años (media 61 años). Todos ellos en programa estable de hemodiálisis, y buen acceso vascular (19 fístulas arteriovenosa y 2 catéteres sin problemas de permeabilidad). La velocidad habitual de la bomba de sangre fue de 350 a $400 \mathrm{ml} / \mathrm{m}$, y el flujo de líquido de diálisis de $700 \mathrm{ml} / \mathrm{m}$.

Se recogen las determinaciones analíticas mensual de $\mathrm{Kt} / \mathrm{v}$ y $\mathrm{Hb}$, de todos los pacientes durante el año previo al inicio con HDFPO y durante todo el primer año de terapia con HDFP0. Para la evaluación del los requerimientos de eritropoyetina, se procede a la cuantificación por sesión de cada uno de los pacientes, igualmente durante todo el año previo al inicio de la HDFP0, y todo el año de ya tratamiento. Los resultados se expresan como media aritmética y desviación típica. Para los datos cuantitativos paramétricos ( $\mathrm{Kt} / \mathrm{v}$ y Hb), se ha empleado la t de Student, para un nivel de significación $\mathrm{P}<0.05$ y un nivel de confianza del $95 \%$. Para los datos no paramétricos (determinación dosis eritropoyetina) se ha utilizado la W de Wilcoxon, diferenciando dos tipos de eritropoyetina. La evaluación de la calidad de vida 
se realiza mediante una encuesta a los pacientes en la que se contemplaron dos bloques: tolerancia al tratamiento y bienestar general, sus resultados se midieron mediante porcentajes.

\section{Resultados}

Respecto al Kt/v, el 90,7\% de nuestros pacientes han aumentado su media en un 10,5\%. Sólo un 9,3\% de pacientes a los cuales se les había reducido el tiempo de diálisis bajaron su media de $\mathrm{Kt} / \mathrm{v}$ un $6,12 \%$ ( $\sin$ que estas fueran inferiores a 1,4 ). Respecto a la hemoglobina, no existe una variación significativa entre los niveles de hemoglobina entre las dos terapias, manteniéndose la media por paciente entre 11,4 y 11,2 con un rango de $+/-0,12$ y una diferencia de las medias igual a 0 .

En cuanto a la dosis de eritropoyetina (figura 1) observamos que más del $57 \%$ de los pacientes que han pasado de HDAF a HDFPO, han visto reducidas sus necesidades de eritropoyetina en un $39 \%$ (tabla 1).

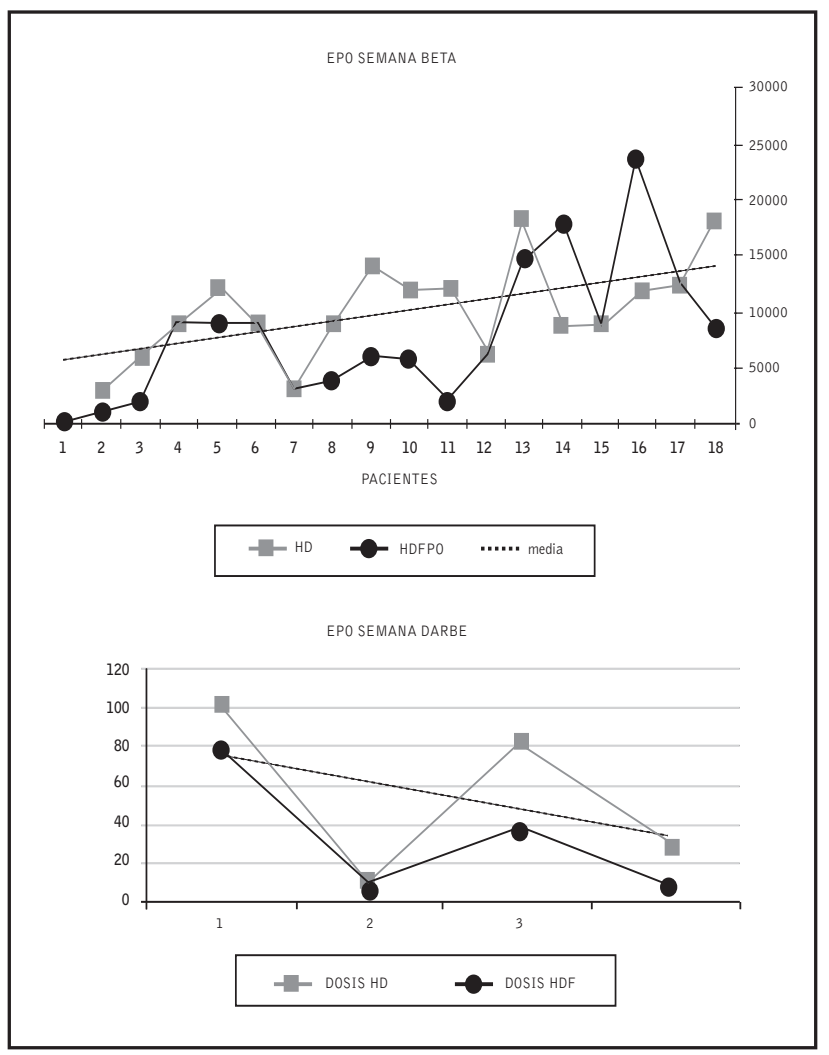

Figura 1. Necesidades de dosis de eritropoyetina

\begin{tabular}{|c|c|c|}
\hline Reducen Epo & Mantienen Epo & Aumentan Epo \\
\hline $57,10 \%$ & $33,33 \%$ & $9,50 \%$ \\
\hline
\end{tabular}

Tabla 1. Porcentaje de pacientes que reducen la dosis de eritropoyetina

Los resultados de la encuesta sobre calidad de vida se muestran en la tabla 2. Nos sorprendió que más del $50 \%$ de nuestros pacientes manifiesten la misma sensación de sed cuando se espera que ésta disminuya con la terapia on-line, lo mismo nos ocurre con el apetito, donde más del $60 \%$ dice tener el mismo apetito cuando lo esperado es que aumente. Las posibles causas de estos resultados podrían ser:

\begin{tabular}{|c|c|c|c|}
\hline TOLERANCIA & MEJOR & IGUAL & PEOR \\
\hline $\begin{array}{l}\text { ¿Cómo pasa las diálisis desde que } \\
\text { esta realizando la nueva terapia? }\end{array}$ & $71 \%$ & $23 \%$ & $4,76 \%$ \\
\hline Las rampas son & $67 \%$ & $23,50 \%$ & $9,50 \%$ \\
\hline Durante la diálisis mantiene la T/A & $34 \%$ & $47,50 \%$ & $18,50 \%$ \\
\hline \multicolumn{4}{|l|}{ BIENESTAR } \\
\hline $\begin{array}{l}\text { Desde que dializa con este sistema } \\
\text { se encuentra en relación al } \\
\text { cansancio }\end{array}$ & $62 \%$ & $28,50 \%$ & $9,50 \%$ \\
\hline En relación a la sed que siente & $23,90 \%$ & $57,10 \%$ & $19 \%$ \\
\hline En relación con el apetito nota & $28,50 \%$ & $62 \%$ & $9,50 \%$ \\
\hline ¿Ha reducido los hipotensores? & $28,60 \%$ & $71,40 \%$ & $0,00 \%$ \\
\hline $\begin{array}{l}\text { ¿Ha reducido el tiempo de } \\
\text { hemodiálisis? }\end{array}$ & $43 \%$ & $52,50 \%$ & $4,70 \%$ \\
\hline $\begin{array}{l}\text { En general desde que ha iniciado la } \\
\text { nueva terapia, se encuentra }\end{array}$ & $66,60 \%$ & $33,30 \%$ & $10,00 \%$ \\
\hline
\end{tabular}

Tabla 2. Resultados de la encuesta

- Respecto a que no disminuya la sensación de sed: uno de los aspectos que más inciden en la sensación de sed es el aumento de $\mathrm{Na}^{+}$en el baño, pero en la HDFPO las únicas modificaciones en relación al $\mathrm{Na}^{+}$han sido en sentido descendente, por tanto, la sensación de sed debería disminuir.

- En cuanto al apetito, ya que determinados parámetros analíticos reflejan el grado de nutrición y al haber mejorado la PCR y la albúmina en los pacientes tratados con HDFPO se deduce que están mejor nutridos, además mantienen los niveles de hemoglobina con dosis más bajas de eritropoyetina.

Más del $50 \%$ de los pacientes que son trasgresores de las recomendaciones en cuanto a la ingesta de líquidos 
y alimentos, y estos hábitos, no se han modificado en ninguna de las dos terapias; esto justificaría los resultados en cuanto la sensación de hambre y sed. Por tanto dividimos a los pacientes en transgresores o no, teniendo en cuenta el incremento de peso interdiálisis y la respuesta a la pregunta: ¿Come y bebe usted lo que le apetece o bien procura mantenerse dentro de unos límites de dieta y líquido? Observamos que 9 pacientes manifestaban ser disciplinados en cuanto al aumento de peso y 12 no serlo. La relación de ambos grupos y el aumento de peso se refleja en la tabla 3. Verificamos que existe correlación entre ser transgresor y manifestar la misma sensación de hambre y sed.

\begin{tabular}{|l|c|c|}
\hline \multicolumn{1}{|c|}{ GRUPO } & HD convencional & HDFPO \\
\hline $\begin{array}{l}\text { Disciplinados } \\
9(42,8 \%)\end{array}$ & $+/-2,5 \mathrm{Kgs}$ & $1,5 \mathrm{Kg}+/-500 \mathrm{~g}$ \\
\hline $\begin{array}{l}\text { No discipliandos } \\
12(57,2 \%)\end{array}$ & $+/-4,5 \mathrm{Kg}$ & $3 \mathrm{Kg}+/-500 \mathrm{~g}$ \\
\hline
\end{tabular}

Tabla 3. Incremento de peso interdiálisis de los pacientes transgresores o no transgresores

\section{Discusión}

Los resultados obtenidos en este trabajo son similares a los de otros estudios ${ }^{4-5}$. Se verifica una mejora sustancial y significativa en las determinaciones de $\mathrm{Kt} / \mathrm{v}$, además de verse reducida de forma más que considerable, un $39 \%$ en más del $57 \%$ de los pacientes, las necesidades de eritropoyetina para conseguir valores similares de hemoglobina. Sin embargo, y a diferencia con otros estudios realizados ${ }^{5-6}$, existen notables diferencias en la percepción de bienestar de los pacientes, en lo que se refiere a las sensaciones de hambre y sed. Era previsible, que con el cambio de una terapia a otra los mismos pacientes presentaran un mayor apetito $y$ menos sed. A pesar de las percepciones de los pacientes y teniendo en cuenta sus características respecto a hábitos dietéticos, y el incremento de peso ínter diálisis, se observa claramente que hay una descenso del incremento de peso, lo que nos revelaría que a pesar de esta percepción, para nosotros atribuible al habito de una dieta poco adecuada bajo las dos forma de tratamiento parece que estos pacientes en realidad comen más porque tienen más apetito, $\mathrm{y}$ beben menos. Por tanto, pensamos que ya que ha mejorado el $\mathrm{Kt} / \mathrm{v}$ y disminuido las necesidades de EPO y se observa un mejor estado general de los pacientes y se sienten mejor, la HDFPO se demuestra una técnica beneficiosa para pacientes tanto cumplidores como transgresores de la dieta.

\section{Bibliografia}

1. Canaud B, Kerr P, Argiles A, Flavier JL, Stec F, Mijon $C$. Is hemodiafiltration the dialsysis moadallity of choice for the next decade? Kidney Int. 1993;43 (sup 41):s296-s299.

2. García $H$, Hernández-Jaras J, Maduell $F$, Yago M, Calvo C, Navarro V, Villatoro J. Eficacia de la hemodiafiltración en línea comparada con la hemodiálisis de alto flujo. Nefrología. 1998; 18:476-482.

3. Manduell F, García H, Calvo C, Navarro V, Comparación de la infusión predilucional versus posdilucioal en HDF en línea. Nefrología. 1998; 18 supl. 3-49.

4. Bonforte G, Grillo P, Zerbi S, Suriam M. Improvement of anemia in hemodiálisis patients treated by hemodiafiltration with high-voume on-line prepared subtition fluid. Blood Purif. 2002; 20:357-63.

5. Pedrini LA. On- line hemodiafiltration: technique and efficiency. J Nephrol. 2003; 16(Supl.7); 557-563.

6. Mucsi I, Molnar MZ, Ambrus C, Sceifer L, Kovecs $A Z$, Zorrer $R$, et al. Retsless legs syndrome, insomnia and quality life in patients on maintenance dialysis. Nephrol Dial Transplant. 2005; 20: 571-7. 